Article

\title{
The 1st Law of Thermodynamics for the Mean Energy of a Closed Quantum System in the Aharonov-Vaidman Gauge
}

\author{
Allen D. Parks \\ Electromagnetic and Sensor Systems Department, 18444 Frontage Road Suite 327, \\ Naval Surface Warfare Center Dahlgren Division, Dahlgren, VA 22448-5161, USA; \\ E-Mail: allen.parks@navy.mil; Tel.:+1-540-419-3777 \\ Academic Editor: Palle E. T. Jorgensen
}

Received: 18 February 2015 / Accepted: 19 May 2015 / Published: 1 June 2015

\begin{abstract}
The Aharonov-Vaidman gauge additively transforms the mean energy of a quantum mechanical system into a weak valued system energy. In this paper, the equation of motion of this weak valued energy is used to provide a mathematical statement of an extended 1st Law of Thermodynamics that is applicable to the mean energy of a closed quantum system when the mean energy is expressed in the Aharonov-Vaidman gauge, i.e., when the system's energy is weak valued. This is achieved by identifying the generalized heat and work exchange terms that appear in the equation of motion for weak valued energy. The complex valued contributions of the additive gauge term to these generalized exchange terms are discussed and this extended 1st Law is shown to subsume the usual 1st Law that is applicable for the mean energy of a closed quantum system. It is found that the gauge transformation introduces an additional energy uncertainty exchange term that - while it is neither a heat nor a work exchange term-is necessary for the conservation of weak valued energy. A spin-1/2 particle in a uniform magnetic field is used to illustrate aspects of the theory. It is demonstrated for this case that the extended 1st Law implies the existence of a gauge potential $\omega$ and that it generates a non-vanishing gauge field $F$. It is also shown for this case that the energy uncertainty exchange accumulated during the evolution of the system along a closed evolutionary cycle $C$ in an associated parameter space is a geometric phase. This phase is equal to both the path integral of $\omega$ along $C$ and the integral of the flux of $F$ through the area enclosed by $C$.
\end{abstract}


Keywords: Aharonov-Vaidman gauge; weak values; 1st Law of Thermodynamics; closed quantum system; energy conservation; principal fiber bundle; geometric phase; gauge potential; gauge field; energy uncertainty exchange

\section{Introduction}

State pre-selection and post-selection (PPS) techniques have been used in recent years to manipulate and control quantum systems in such diverse research areas as quantum system-environment interactions (e.g., [1]), the quantum eraser (e.g., [2]), and Pancharatnam phase (e.g., [3,4]). An especially important PPS application area is the time symmetric reformulation of quantum mechanics [5] and the closely related notion of the weak value of a quantum mechanical observable (e.g., [6-8]).

The weak value $A_{w}$ of a quantum mechanical observable $A$ is the statistical result of a standard measurement procedure performed upon a PPS ensemble of quantum systems when the interaction between the measurement apparatus and each system is sufficiently weak. Unlike a standard strong measurement of $A$, which significantly disturbs the measured system and yields the mean value $\langle A\rangle$ of the associated operator $\hat{A}$ as the measured value of the observable, a weak measurement of $A$ performed upon a PPS system does not appreciably disturb the system and yields $A_{w}$ as the measured value of $A$. Unlike $\langle A\rangle$-which is real valued and bounded by the eigenvalue spectral limits of $\hat{A}-A_{w}$ can exhibit such eccentric properties as being complex valued and having its real part greatly exceed $\hat{A}$ 's spectral limits. While the interpretation of weak values remains somewhat controversial, several of the unusual properties predicted by weak value theory have been experimentally verified (e.g., [9-13]).

Much attention has also been devoted to studying the intersection between thermodynamics and quantum mechanics. Such research includes a substantial body of literature discussing-for example-quantum heat engines (e.g., [14-18]), thermodynamics of open quantum systems (e.g., [19]), entanglement and work (e.g., [20]), quantum thermometry and heat baths (e.g., [21,22]), quantum refrigerators (e.g., [23,24]), Rényi entropy flow [25], qubit and qutrit work extraction (e.g., [26,27]), and quantum measurement control of thermodynamics [28].

Recently, a PPS defined uncertainty quantity called the Aharonov-Vaidman (AV) gauge was introduced as a new "scale of measurement" for the mean values of quantum mechanical observables [29]. This gauge additively transforms a mean value of an observable into an associated weak value and induces each of the eccentric characteristics exhibited by weak values. Since the total time derivative of the AV gauged mean value yields the equation of motion for the corresponding weak value, both the weak value and its equation of motion can be considered to be the associated mean value and its equation of motion expressed in the AV gauge.

The purpose of this paper is to further examine the thermodynamics/quantum mechanics intersection from the perspective of AV gauge theory. In particular, by identifying heat and work exchange terms which appear in the equation of motion for the mean energy of a closed quantum system when the mean energy is expressed in the AV gauge, an extended version of the 1st Law of Thermodynamics that applies to weak valued energy systems and subsumes the 1st Law for closed 
mean energy systems is obtained. A peculiar energy uncertainty exchange term which is neither a heat nor a work exchange term - but is nonetheless required for weak valued energy conservation-is shown to be induced by the gauge transformation. The extended 1st Law is illustrated via its application to a spin-1/2 particle in a uniform magnetic field. For this system it is shown that: $(i)$ the extended 1st Law implies the existence of a gauge potential and an associated non-vanishing gauge field; and (ii) the energy uncertainty exchange accumulated during a cyclic evolution of the system in a requisite parameter space is a geometric phase. The value of this phase acquired along a closed evolutionary cycle $C$ of the system in this parameter space is equal to the path integral of the gauge potential along $C$ and - equivalently - it is also equal to the integral of the gauge field flux through the area enclosed by $C$.

The remainder of this paper is organized as follows: a brief overview of the AV gauge for energy is presented in the next section. The general theory of the extended 1st Law is derived in Section 3 and is applied to a spin-1/2 particle in a uniform magnetic field in Section 4. The theory of the weak valued energy gauge potential and gauge field for a spin-1/2 particle in a uniform magnetic field is developed in Section 5 and the associated geometric phase is introduced and discussed in Section 6. Closing remarks comprise the final section of this paper.

\section{The AV Gauge for Energy}

A quantum mechanical Hamiltonian operator $\widehat{H}$ acting upon a pre-selected energy state $\left|\psi_{i}\right\rangle$ at time $t$ can be uniquely expressed as [8]

$$
\widehat{H}\left|\psi_{i}\right\rangle=\langle H\rangle\left|\psi_{i}\right\rangle+\Delta H\left|\psi_{i}^{\perp}\right\rangle
$$

where $\langle H\rangle=\left\langle\psi_{i}|\widehat{H}| \psi_{i}\right\rangle$ is the mean energy and $\Delta H=\sqrt{\left\langle H^{2}\right\rangle-\langle H\rangle^{2}}$ is the energy uncertainty. The state $\left|\psi_{i}^{\perp}\right\rangle$ is $\left|\psi_{i}\right\rangle$ 's orthogonal companion state at $t$ that belongs to the subspace of $\widehat{H}$ 's Hilbert space $\mathscr{H}$ that is the orthogonal complement of the subspace of $\mathscr{H}$ that contains $\left|\psi_{i}\right\rangle$. This companion state satisfies the conditions

$$
\left\langle\psi_{i}^{\perp} \mid \psi_{i}\right\rangle=0 \text { and } \Delta H=\left\langle\psi_{i}^{\perp}|\widehat{H}| \psi_{i}\right\rangle
$$

The AV gauge is a consequence of the second term on the right-hand side of Equation (1). More specifically, it is obtained when both sides of Equation (1) are multiplied from the left by the post-selection bra state $\left\langle\psi_{f}\right|$ at time $t$ and then divided by the scalar product $\left\langle\psi_{f} \mid \psi_{i}\right\rangle \neq 0$. This yields the following identity for the weak valued energy $H_{w}$ at $t$ :

$$
H_{w} \equiv \frac{\left\langle\psi_{f}|\widehat{H}| \psi_{i}\right\rangle}{\left\langle\psi_{f} \mid \psi_{i}\right\rangle}=\langle H\rangle+\Omega
$$

Thus, $H_{w}$ is related to $\langle H\rangle$ by an additive transformation-the AV transformation in gauge $\left\langle\psi_{f}\right|$ (or - more simply - the AV gauge transformation) — of $\langle H\rangle$. The additive term

$$
\Omega \equiv \Delta H \frac{\left\langle\psi_{f} \mid \psi_{i}^{\perp}\right\rangle}{\left\langle\psi_{f} \mid \psi_{i}\right\rangle}=\Delta H \cdot \beta
$$

is the associated $A V$ gauge for energy. 
Equations (3) and (4) show that weak valued energy generalizes the notion of mean energy in the sense that $H_{w}$ can be considered to be $\langle H\rangle$ expressed in AV gauge $\left\langle\psi_{f}\right|$. Thus, from a measurement perspective, an apparatus that measures $H_{w}$ actually measures $\langle H\rangle$ in an AV gauge (the special AV gauge $\left\langle\psi_{f}\right|=\left\langle\psi_{i}\right|$ is called the mean gauge since $\Omega=0$ and $H_{w}=\langle H\rangle$ ). It is also clear from these equations that the gauge term $\Omega$ is solely responsible for any eccentric properties associated with the weak valued energy, i.e., $H_{w}$ is complex valued only when $\Omega$ is complex valued and $R e H_{w}$ can exceed the eigen-energy spectral limits of $\widehat{H}$ only when $\left\langle\psi_{f}\right| \rightarrow\left\langle\psi_{i}^{\perp}\right|$ since in this case $|\operatorname{Re} \Omega| \rightarrow \infty$.

In summary, $H_{w}$ is an additive blending of the standard properties of $\langle H\rangle$ with the non-standard eccentric properties introduced by $\Omega$. Consequently, $H_{w}$ completely subsumes the properties of $\langle H\rangle$-regardless of the choice of gauge. This is also the case for $H_{w}$ 's equation of motion. To see this, assume that $H_{w}$ is time dependent and simply observe that the equation of motion for $H_{w}$ is given by the total time derivative of Equation (3), i.e.,

$$
\dot{H}_{w}=\langle\dot{H}\rangle+\dot{\Omega}=\langle\dot{H}\rangle+(\dot{\Delta H}) \beta+\Delta H(\dot{\beta})
$$

Thus, the equation of motion for $H_{w}$ subsumes that for $\langle H\rangle$ and can be viewed as an AV gauge transformation of $\langle\dot{H}\rangle$ by $\dot{\Omega}$.

\section{The Extended 1st Law}

In this section, the results and notation of Section 2 are used to develop a mathematical statement of an extended version of the $1^{\text {st }}$ Law of Thermodynamics that applies to a closed quantum system when the system's mean energy is expressed in an AV gauge. To this end, consider a closed quantum system described by a time dependent Hamiltonian $\widehat{H}$ and let

$$
\begin{aligned}
& \left|\psi_{i}\right\rangle=\sum_{J} a_{j}\left|\varepsilon_{j}\right\rangle \\
& \left|\psi_{f}\right\rangle=\sum_{J} b_{j}\left|\varepsilon_{j}\right\rangle
\end{aligned}
$$

and

$$
\left|\psi_{i}^{\perp}\right\rangle=\sum_{J} c_{j}\left|\varepsilon_{j}\right\rangle
$$

be time dependent energy states, where $\widehat{H}\left|\varepsilon_{j}\right\rangle=\varepsilon_{j}\left|\varepsilon_{j}\right\rangle$ and $J$ is an eigen-energy indexing set. Using these, the results in Section 2, and setting $K=J$, it is found that

$$
\begin{gathered}
\langle\dot{H}\rangle=\sum_{J}\left(a_{J}^{*} a_{J}\right) \varepsilon_{j}+\sum_{J}\left(a_{j}^{*} a_{j}\right) \dot{\varepsilon}_{j} \\
\Delta H=\left[\sum_{J} a_{j}^{*} a_{j} \varepsilon_{j}^{2}-\left(\sum_{J} a_{j}^{*} a_{j} \varepsilon_{j}\right)^{2}\right]^{1 / 2} \\
(\dot{\Delta H})=\frac{1}{2 \Delta H}\left[\sum_{J}\left(a_{J}^{*} a_{J}\right) \varepsilon_{j}\left(\varepsilon_{j}-2 \sum_{K} a_{k}^{*} a_{k} \varepsilon_{k}\right)+2 \sum_{J} a_{j}^{*} a_{j} \varepsilon_{j}\left(\dot{\varepsilon}_{j}-\sum_{K} a_{k}^{*} a_{k} \dot{\varepsilon}_{k}\right)\right] \\
\beta=\frac{\sum_{J} b_{j}^{*} c_{j}}{\sum_{J} b_{j}^{*} a_{j}} \equiv \frac{X_{c}}{X_{a}}
\end{gathered}
$$


and

$$
\dot{\beta}=\frac{\left(X_{a}^{2}\right)^{*}\left[X_{a} \dot{X}_{c}-\dot{X}_{a} X_{c}\right]}{\left(X_{a}^{2}\right)^{*}\left(X_{a}^{2}\right)}
$$

where

$$
\dot{X}_{\mu}=\sum_{J} b_{j}^{*} \mu_{j}\left[\frac{\left(b_{j}^{*} b_{J}\right)}{\left(b_{j}^{*} b_{j}\right)}+\frac{\left(\mu_{j}^{*} \mu_{J}\right)}{\left(\mu_{j}^{*} \mu_{j}\right)}-\frac{\dot{b}_{j}}{b_{j}}-\frac{\dot{\mu}_{j}^{*}}{\mu_{j}^{*}}\right], \mu \in\{a, c\}
$$

After multiplying both sides of Equations (5)-(9) by the time infinitesimal $d t$; substituting the resulting expressions for $\langle\dot{H}\rangle d t=d\langle H\rangle,(\Delta \dot{H}) d t=d(\Delta H), \dot{\beta} d t=d \beta$, and $\dot{X}_{\mu} d t=d X_{\mu}$ into the right-hand side of $\dot{H}_{w} d t=d H_{w}$; and identifying and collecting together those terms containing infinitesimal changes in probability (i.e., $\left.d\left(x_{j}^{*} x_{j}\right), x \in\{a, b, c\}\right)$ and those terms containing infinitesimal changes $d \varepsilon_{j}$ in eigen-energy as heat and work exchange terms (e.g., [30]), respectively, the following mathematical statement of the extended 1st Law of Thermodynamics for the mean energy of a closed quantum system in the AV gauge is obtained:

$$
d H_{w}=d Q_{w}+d W_{w}-d \mathcal{J}_{w}
$$

where (subscripts " $R$ " and "I" denote the real and imaginary parts of a complex valued quantity)

$$
d Q_{w}=d Q+d Q_{R}+i d Q_{I}
$$

is the complex valued heat exchange term,

$$
d W_{w}=d W+d W_{R}+i d W_{I}
$$

is the complex valued work exchange term, and

$$
d \mathcal{J}_{w}=d \mathcal{J}_{R}+i d \mathcal{J}_{I}
$$

is the complex valued energy uncertainty exchange term. Here

$$
\begin{gathered}
d Q=\sum_{J} \varepsilon_{j} d\left(a_{j}^{*} a_{j}\right) \\
d W=\sum_{J}\left(a_{j}^{*} a_{j}\right) d \varepsilon_{j} \\
d Q_{R}=\frac{\operatorname{Re}\left(X_{a}^{*} X_{c}\right)}{2 \Delta H\left|X_{a}\right|^{2}} \sum_{J} \varepsilon_{j}\left(\varepsilon_{j}-2 \sum_{K} a_{k}^{*} a_{k} \varepsilon_{k}\right) d\left(a_{j}^{*} a_{j}\right) \\
+\Delta H \sum_{J}\left\{\left[A \operatorname{Re}\left(b_{j}^{*} c_{j}\right)-C \operatorname{Im}\left(b_{j}^{*} c_{j}\right)\right]\left[\frac{d\left(b_{j}^{*} b_{j}\right)}{b_{j}^{*} b_{j}}+\frac{d\left(c_{j}^{*} c_{j}\right)}{c_{j}^{*} c_{j}}\right]\right. \\
\left.-\left[D \operatorname{Re}\left(b_{j}^{*} a_{j}\right)-E \operatorname{Im}\left(b_{j}^{*} a_{j}\right)\right]\left[\frac{d\left(b_{j}^{*} b_{j}\right)}{b_{j}^{*} b_{j}}+\frac{d\left(a_{j}^{*} a_{j}\right)}{a_{j}^{*} a_{j}}\right]\right\} \\
+\Delta H \sum_{I}\left\{\left[\operatorname{Im}\left(X_{a}^{*} X_{c}\right) \sum_{J} \varepsilon_{j}\left(\varepsilon_{j}-2 \sum_{K} \operatorname{Im}\left(b_{j}^{*} c_{j}\right)+C \operatorname{Re}\left(b_{j}^{*} c_{j}\right)\right]\left[\frac{d\left(b_{j}^{*} b_{j}\right)}{b_{j}^{*} b_{j}}+\frac{d\left(c_{j}^{*} c_{j}\right)}{c_{j}^{*} c_{j}}\right]\right.\right. \\
\left.-\left[D \operatorname{Im}\left(b_{j}^{*} a_{j}\right)+E \operatorname{Re}\left(b_{j}^{*} a_{j}\right)\right]\left[\frac{d\left(b_{j}^{*} b_{j}\right)}{b_{j}^{*} b_{j}}+\frac{d\left(a_{j}^{*} a_{j}\right)}{a_{j}^{*} a_{j}}\right]\right\}
\end{gathered}
$$




$$
\begin{gathered}
d W_{R}=\frac{\operatorname{Re}\left(X_{a}^{*} X_{c}\right)}{\Delta H\left|X_{a}\right|^{2}} \sum_{J} a_{j}^{*} a_{j} \varepsilon_{j}\left(d \varepsilon_{j}-\sum_{K} a_{k}^{*} a_{k} d \varepsilon_{k}\right) \\
d W_{I}=\frac{\operatorname{Im}\left(X_{a}^{*} X_{c}\right)}{\Delta H\left|X_{a}\right|^{2}} \sum_{J} a_{j}^{*} a_{j} \varepsilon_{j}\left(d \varepsilon_{j}-\sum_{K} a_{k}^{*} a_{k} d \varepsilon_{k}\right) \\
d \mathcal{J}_{R}=\Delta H\left[A \operatorname{Re}\left(d \mathcal{J}_{c}\right)-C \operatorname{Im}\left(d \mathcal{J}_{c}\right)-D \operatorname{Re}\left(d \mathcal{J}_{a}\right)+E \operatorname{Im}\left(d \mathcal{J}_{a}\right)\right]
\end{gathered}
$$

and

$$
d \mathcal{J}_{I}=\Delta H\left[A \operatorname{Im}\left(d \mathcal{J}_{c}\right)+C \operatorname{Re}\left(d \mathcal{J}_{c}\right)-D \operatorname{Im}\left(d \mathcal{J}_{a}\right)-E \operatorname{Re}\left(d \mathcal{J}_{a}\right)\right]
$$

where

$$
\begin{gathered}
d \mathcal{J}_{\mu}=\sum_{J} b_{j}^{*} \mu_{j}\left[\frac{d b_{j}}{b_{j}}+\frac{d \mu_{j}^{*}}{\mu_{j}^{*}}\right], \mu \in\{a, c\} \\
A=\frac{\operatorname{Re}\left(X_{a}^{2}\right) \operatorname{Re}\left(X_{a}\right)+\operatorname{Im}\left(X_{a}^{2}\right) \operatorname{Im} X_{a}}{\left[\operatorname{Re}\left(X_{a}^{2}\right)\right]^{2}+\left[\operatorname{Im}\left(X_{a}^{2}\right)\right]^{2}} \\
C=\frac{\operatorname{Re}\left(X_{a}^{2}\right) \operatorname{Im}\left(X_{a}\right)-\operatorname{Im}\left(X_{a}^{2}\right) \operatorname{Re}\left(X_{a}\right)}{\left[\operatorname{Re}\left(X_{a}^{2}\right)\right]^{2}+\left[\operatorname{Im}\left(X_{a}^{2}\right)\right]^{2}} \\
D=\frac{\operatorname{Re}\left(X_{a}^{2}\right) \operatorname{Re}\left(X_{c}\right)+\operatorname{Im}\left(X_{a}^{2}\right) \operatorname{Im}\left(X_{c}\right)}{\left[\operatorname{Re}\left(X_{a}^{2}\right)\right]^{2}+\left[\operatorname{Im}\left(X_{a}^{2}\right)\right]^{2}}
\end{gathered}
$$

and

$$
E=\frac{\operatorname{Re}\left(X_{a}^{2}\right) \operatorname{Im}\left(X_{c}\right)-\operatorname{Im}\left(X_{a}^{2}\right) \operatorname{Re}\left(X_{c}\right)}{\left[\operatorname{Re}\left(X_{a}^{2}\right)\right]^{2}+\left[\operatorname{Im}\left(X_{a}^{2}\right)\right]^{2}}
$$

It is obvious from this that the extended 1 st Law subsumes the 1 st Law for mean energy systems since $d H_{w}=d Q+d W=d\langle H\rangle$ when the AV gauge is the mean gauge.

The theory also shows that there are several peculiar thermodynamic properties associated with weak valued energy systems when $\Omega \neq 0$. The most obvious of these is the fact that the exchange terms can be complex valued. This is clearly expected since $\Omega$ can be complex valued. Perhaps the most peculiar property is that energy uncertainty is used and exchanged by weak valued energy systems. In particular, observe that $d W_{x}, x \in\{R, I\}$, varies inversely with energy uncertainty $\Delta H$. This suggests that systems for which $\Delta H$ is small can perform more work than those for which $\Delta H$ is large. Also note that $d \mathcal{J}_{x}, x \in\{R, I\}$, varies directly with $\Delta H$ since the factors in brackets are dimensionless scale factors. This direct variation of $d \mathcal{J}_{w}$ with $\Delta H$ is the reason $d \mathcal{J}_{w}$ is herein called the "energy uncertainty exchange term" and - as will be shown in Section 6- this term can produce a geometric phase (or, equivalently, it can contribute to the strength of an associated gauge field) in weak valued energy systems. Finally, the fact that the first and second summations in $d Q_{x}, x \in\{R, I\}$, vary inversely and directly with $\Delta H$, respectively, indicates there is an "ambivalence" in the heat exchange process in weak valued energy systems, i.e., both small and large energy uncertainties can enhance heat exchange.

When the system Hamiltonian, eigen-energies, and pure energy states are known (as is assumed here), then the functional forms for $H_{w}$ and $\langle H\rangle$ are also known. Consequently, both $d H_{w}$ and $d\langle H\rangle$ are exact differentials (recall that $d u$ is exact in a region $\mathcal{R}$ of some space $\mathcal{S}$ if there exists a function $v$ 
defined on $\mathcal{R}$ such that $d u=d v$ ). The following theorem is an obvious consequence of this exactness and is stated without proof.

Theorem 3.1 Suppose $\mathcal{S}$ is a Cartesian space with axes corresponding to quantities that parameterize the variations of $\varepsilon_{j}, a_{j}, b_{j}$, and $c_{j}, j \in J$. If $H_{w}$ is defined on region $\mathcal{R} \subset \mathcal{S}$ and $C$ is a smooth closed cycle in $\mathcal{R}$, then

$$
\oint_{C} d H_{w}=0
$$

Thus, the heat, work, and energy uncertainty exchange terms vary during the evolution along $C$ of a closed quantum system in an AV gauge in precisely the manner required to insure there is no change in the associated weak valued energy-i.e., $\Delta H_{w}=0$ (here " $\Delta X$ " refers to either the change in quantity " $X$ " or the amount of " $X$ " exchanged; the meaning of " $\Delta$ "-i.e., "uncertainty" or "change" - is clear from the context in which it is used). The next two corollaries to this theorem are also stated without proof since they obviously follow from this theorem and the fact that $H_{w}=H_{w_{R}}+i H_{w_{I}}$ is complex valued:

\section{Corollary 3.2}

$$
\oint_{C} d H_{w_{R}}=0=\oint_{C} d H_{w_{I}}
$$

Corollary 3.3 The total work, heat, and energy uncertainty exchanged during an evolution of a weak valued energy system in AV gauge $\left\langle\psi_{f}\right| \neq\left\langle\psi_{i}\right|$ along C conform to the following equalities:

$$
\begin{gathered}
\Delta W+\Delta W_{R}=-\left(\Delta Q+\Delta Q_{R}-\Delta \mathcal{J}_{R}\right) \\
\Delta W_{I}=-\left(\Delta Q_{I}-\Delta \mathcal{J}_{I}\right)
\end{gathered}
$$

Note that, for mean gauge systems, this theorem and its corollaries also apply to mean energy systems. In this case, since $d H_{w}=d\langle H\rangle$ and $\Delta W_{x}=\Delta Q_{x}=\Delta \mathcal{J}_{x}=0, x \in\{R, I\}$, then

$$
\oint_{C} d\langle H\rangle=0
$$

and

$$
\Delta W=-\Delta Q
$$

\section{The Extended 1st Law for a Spin-1/2 Particle in a Uniform Magnetic Field}

In order to illustrate aspects of the theory developed above, consider a spin- $1 / 2$ particle with magnetic moment $\gamma$ under the influence of a magnetic field $\boldsymbol{B}(t)$ that is assumed to be uniform at $t$ and is oriented along the positive $z$ axis of a three dimensional Cartesian reference frame. The Hamiltonian for this system is

$$
\widehat{H}(t)=-\gamma B(t) \hat{\sigma}_{z}
$$

where $\hat{\sigma}_{z}$ is a Pauli spin operator and 


$$
\widehat{H}(t)| \pm \varepsilon(t)\rangle= \pm \varepsilon(t)| \pm \varepsilon(t)\rangle
$$

Here $\pm \varepsilon(t)=\mp \gamma B(t)$ are the system's energy eigenvalues and $| \pm \varepsilon(t)\rangle$ are the associated orthonormal eigen-states (i.e., $\langle \pm \varepsilon(t) \mid \mp \varepsilon(t)\rangle=0$ and $\langle \pm \varepsilon(t) \mid \pm \varepsilon(t)\rangle=1$ ).

Let the time dependent angle $\theta(t)$ and the fixed angle $\varphi$ parameterize the mixing of superposed energy eigen-states when forming the PPS and orthogonal companion states. For the sake of simplicity choose

$$
\begin{gathered}
\left|\psi_{i}\right\rangle=\sin \theta(t)|+\varepsilon(t)\rangle+\cos \theta(t)|-\varepsilon(t)\rangle \\
\left|\psi_{f}\right\rangle=\sin \varphi|+\varepsilon(t)\rangle+\cos \varphi|-\varepsilon(t)\rangle
\end{gathered}
$$

and

$$
\left|\psi_{i}^{\perp}\right\rangle=-\cos \theta(t)|+\varepsilon(t)\rangle+\sin \theta(t)|-\varepsilon(t)\rangle
$$

so that the imaginary parts of all exchange terms vanish. Note that these states are pure and normalized; the AV gauge of the system is $\left\langle\psi_{f}\right| \neq\left\langle\psi_{i}\right| ;\left\langle\psi_{f} \mid \psi_{i}\right\rangle \neq 0$ when $\theta(t) \neq \varphi$; and-as required $-\left\langle\psi_{i} \mid \psi_{i}^{\perp}\right\rangle=0$ and $\Delta H=\left\langle\psi_{i}^{\perp}|\widehat{H}| \psi_{i}\right\rangle$.

From this, the following identifications can be made with quantities appearing in the extended 1st Law theory developed in Section 3 (hereafter the time dependence of $\theta, B$, and $\widehat{H}$ will not be shown):

$$
\begin{gathered}
\varepsilon_{1}=+\varepsilon \quad \varepsilon_{2}=-\varepsilon \\
a_{1}=\sin \theta \quad a_{2}=\cos \theta \\
b_{1}=\sin \varphi \quad b_{2}=\cos \varphi
\end{gathered}
$$

and

$$
c_{1}=-\cos \theta \quad c_{2}=+\sin \theta
$$

It follows that

$$
\begin{gathered}
\Delta H=\gamma B \sin 2 \theta \\
X_{a}=\cos (\theta-\varphi) \\
X_{c}=+\sin (\theta-\varphi) \\
A=\frac{1}{\cos (\theta-\varphi)} \\
D=+\frac{\tan (\theta-\varphi)}{\cos (\theta-\varphi)}
\end{gathered}
$$

and

$$
C=0=E
$$

in which case

$$
\begin{gathered}
d Q=-4 \gamma B \sin \theta \cos \theta d \theta \\
d Q_{R}=\gamma B\left[2 \cos 2 \theta \tan (\theta-\varphi)+2 \sin 2 \theta \sec ^{2}(\theta-\varphi)\right] d \theta
\end{gathered}
$$




$$
\begin{gathered}
d W=\gamma\left[\cos ^{2} \theta-\sin ^{2} \theta\right] d B \\
d W_{R}=\gamma \sin 2 \theta \tan (\theta-\varphi) d B \\
d \mathcal{J}_{R}=\gamma B \sin 2 \theta \sec ^{2}(\theta-\varphi) d \theta
\end{gathered}
$$

and

$$
d Q_{I}=d W_{I}=d \mathcal{J}_{I}=0
$$

Thus, for this system the extended $1^{\text {st }}$ Law can be stated as

$$
\begin{aligned}
& d H_{w}=\gamma B\left[-4 \sin \theta \cos \theta+2 \cos 2 \theta \tan (\theta-\varphi)+2 \sin 2 \theta \sec ^{2}(\theta-\varphi)\right] d \theta \\
&+\gamma\left[\cos ^{2} \theta-\sin ^{2} \theta+\sin 2 \theta \tan (\theta-\varphi)\right] d B-\gamma B \sin 2 \theta \sec ^{2}(\theta-\varphi) d \theta
\end{aligned}
$$

where the first and second terms on the right hand side of this expression are the heat and work exchange terms, respectively, and the last term corresponds to the energy uncertainty exchange term.

It is easily verified that this result agrees exactly with that obtained directly from Equations (3)-(4), i.e.,

$$
H_{w}=\langle H\rangle+\Delta H \cdot \beta
$$

where

$$
\langle H\rangle=\gamma B\left[\cos ^{2} \theta-\sin ^{2} \theta\right]
$$

and

$$
\beta=+\tan (\theta-\varphi)
$$

Note that, unlike the general development in Section 3, obtaining the extended 1st Law directly from this expression does not straightforwardly discriminate between heat and energy uncertainty exchange terms since they both depend upon $d \theta$.

\section{The Weak Valued Energy Gauge Field}

Using the results of the last section, define

$$
\begin{gathered}
f \equiv \frac{d Q}{d \theta}=-4 \gamma B \sin \theta \cos \theta \\
f_{\Omega} \equiv \frac{d Q_{R}}{d \theta}=2 \gamma B\left[\cos 2 \theta \tan (\theta-\varphi)+\sin 2 \theta \sec ^{2}(\theta-\varphi)\right] \\
g \equiv \frac{d W}{d B}=\gamma\left[\cos ^{2} \theta-\sin ^{2} \theta\right] \\
g_{\Omega} \equiv \frac{d W_{R}}{d B}=\gamma \sin 2 \theta \tan (\theta-\varphi)
\end{gathered}
$$

and introduce the 1-form

$$
\omega \equiv A_{\theta} d \theta+A_{B} d B
$$

Here $\omega$ is the weak valued energy gauge potential for the spin-1/2 system, where

$$
A_{\theta} \equiv f+f_{\Omega}
$$

and 


$$
A_{B} \equiv g+g_{\Omega}
$$

are recognized as the two components of the associated vector potential (note that $\omega=d H_{w}+d \mathcal{J}_{R}$ ). This will become more clear in the geometric setting of the next section where $\omega$ defines a principle fiber bundle connection.

Let

$$
\mathfrak{B} \equiv\left\{(\theta, B) \in[0,2 \pi) \times\left[0, B_{\max }\right]\right\}
$$

be the region in the real Cartesian plane that is parameterized by the superposition mixing angle $\theta$ and the magnetic field strength $B$, where it is assumed that $B$ is (spatially) uniform at every $(\theta, B) \in \mathfrak{B}$. Since the following theorem is an obvious consequence of Green's theorem and the definitions of $A_{\theta}$ and $A_{B}$, it is stated without proof.

Theorem 5.1 If a closed spin-1/2 system in AV gauge $\left\langle\psi_{f}\right| \neq\left\langle\psi_{i}\right|$ evolves along a closed cycle $C$ in $\mathfrak{B}$ such that the area $\mathcal{A}$ enclosed by $C$ is convex, then

$$
\oint_{C} \omega=\iint_{\mathcal{A}} d \omega=\iint_{\mathcal{A}} F d \theta d B
$$

where

$$
F \equiv \frac{\partial A_{B}}{\partial \theta}-\frac{\partial A_{\theta}}{\partial B}=-\gamma \sin 2 \theta \sec ^{2}(\theta-\varphi)
$$

Here $F$ is the field strength-i.e., the weak valued energy gauge field-derived from the vector potential associated with $\omega$.

Corollary 5.2 The field strength vanishes if a closed spin-1/2 system is in the mean gauge and

$$
\oint_{C} \omega=0
$$

Proof. In the mean gauge $f_{\Omega}=0=g_{\Omega}$ so that $A_{\theta}=f$ and $A_{B}=g$. Since

$$
\frac{\partial A_{B}}{\partial \theta}=\frac{\partial g}{\partial \theta}=-4 \gamma \sin \theta \cos \theta=\frac{\partial f}{\partial B}=\frac{\partial A_{\theta}}{\partial B}
$$

then $F=0$. It follows from this or from Green's theorem that

$$
\oint_{C} \omega=0=\iint_{\mathcal{A}}\left(\frac{\partial A_{B}}{\partial \theta}-\frac{\partial A_{\theta}}{\partial B}\right) d \theta d B
$$

Observe that

$$
d \omega=d A_{\theta} d \theta+d A_{B} d B=d\left(\frac{d Q}{d \theta}+\frac{d Q_{R}}{d \theta}\right) d \theta+d\left(\frac{d W}{d B}+\frac{d W_{R}}{d B}\right) d B=d\left(\frac{d Q_{R}}{d \theta} d \theta+\frac{d W_{R}}{d B} d B\right) \neq 0
$$

where use is made of the fact that $d\left(\frac{d Q}{d \theta} d \theta+\frac{d W}{d B} d B\right)=0$. It follows from this that a non-vanishing gauge field $F$ is generated only when the system is in AV gauge $\left\langle\psi_{f}\right| \neq\left\langle\psi_{i}\right|$ and that it results from a changing vector potential. More specifically, it is produced by changes ("accelerations") in the rates of heat and work exchange with respect to $\theta$ and $B$, respectively. 


\section{The Geometric Phase}

Now consider the principal fiber bundle $P=(\mathfrak{B} \times \mathfrak{R}, p)$ which has as its base space the region $\mathfrak{B}$; as its typical fiber the additive (structure) group $\Re$ of real numbers; the map $p:((\theta, B), r) \mapsto(\theta, B)$ as its bundle projection (in this geometric setting, $F$ is the curvature of the connection $\omega$ on $P$, and $\mathfrak{R}$ corresponds to the gauge symmetry group); and define the 1 form

$$
\omega_{P} \equiv A_{\theta} d \theta+A_{B} d B+d r
$$

which is clearly a connection on $P$. Let $\sigma:[0,1] \rightarrow \mathfrak{B}$ be a smooth curve in $\mathfrak{B}$; $\tilde{\sigma}$ be a horizontal lift of $\sigma$ in $P$ starting at point $\left(\left(\theta_{0}, B_{0}\right), r_{0}\right)$; and

$$
v \equiv \frac{d \theta}{d t} \frac{\partial}{\partial \theta}+\frac{d B}{d t} \frac{\partial}{\partial B}+\frac{d r}{d t} \frac{\partial}{\partial r}
$$

be tangent to $\tilde{\sigma}$. Since $\tilde{\sigma}$ is a horizontal lift, then it must be the case that $\omega_{P}(v)=0$ so that

$$
A_{\theta} \frac{d \theta}{d t}+A_{B} \frac{d B}{d t}+\frac{d r}{d t}=0
$$

or

$$
d r=-\left(A_{\theta} d \theta+A_{B} d B\right)=-\omega
$$

It is clear from the last equation that, in general, the gauge potential $\omega$ governs how the fiber coordinate $r$ changes as $\theta$ and $B$ vary in $\mathfrak{B}$.

Suppose $\sigma$ describes a closed cycle $C$ in $\mathfrak{B}$ such that $\mathcal{A}$ is convex. Then-from Theorem 5.1,

$$
\Delta r=-\oint_{C} \omega=-\iint_{\mathcal{A}} F d \theta d B
$$

Although the cycle $C$ is closed in $\mathfrak{B}$, its horizontal lift is not (in general) closed and returns to the point $\left(\left(\theta_{0}, B_{0}\right), r_{0}+\Delta r\right)$ at the end of the cycle in $\mathfrak{B}$. Since the net change in the fiber coordinate over $C$ is $\Delta r$ (and in general $\Delta r \neq 0$ ), then $\Delta r$ is a geometric phase and the following theorem has been proved (because $\Delta r$ does not depend upon $r_{0}, \Delta r$ is said to be gauge invariant for any choice of gauge $\left.r_{0}\right)$ :

Theorem 6.1 If a closed spin-1/2 system in AV gauge $\left\langle\psi_{f}\right| \neq\left\langle\psi_{i}\right|$ evolves along a closed cycle $C$ in $\mathfrak{B}$ such that the area $\mathcal{A}$ enclosed by $C$ is convex, it accumulates a net geometric phase $\Delta r$ given by

$$
\Delta r=-\oint_{C} \omega=-\iint_{\mathcal{A}} F d \theta d B
$$

when the cycle is completed.

Corollary 6.2 The net geometric phase $\Delta r$ accumulated during a complete cycle $C$ in $\mathfrak{B}$ by a closed spin-1/2 system in the mean gauge is zero.

Proof. This is a trivial consequence of Corollary 5.2.

Theorem 6.3 The net geometric phase $\Delta r$ accumulated by a closed spin-1/2 system in AV gauge $\left\langle\psi_{f}\right| \neq\left\langle\psi_{i}\right|$ during a complete closed cycle $C$ in $\mathfrak{B}$ is 


$$
\Delta r=-\oint_{C} d \mathcal{J}_{R}
$$

Proof. Because

$$
\omega=A_{\theta} d \theta+A_{B} d B=d H_{w}+d \mathcal{J}_{R}
$$

then

$$
d r=-\omega=-\left(d H_{w}+d \mathcal{J}_{R}\right)
$$

and

$$
\Delta r=\oint_{C} d r=-\oint_{C} d H_{w}-\oint_{C} d \mathcal{J}_{R}
$$

Use of the fact that

$$
\oint_{C} d H_{w}=0
$$

completes the proof.

The next two corollaries to this theorem are obvious and are stated without proof for the sake of completeness.

\section{Corollary 6.4}

$$
\oint_{C} d \mathcal{J}_{R}=\iint_{\mathcal{A}} F d \theta d B
$$

\section{Corollary 6.5}

$$
\Delta r=-\gamma \oint_{C} B \sin 2 \theta \sec ^{2}(\theta-\varphi) d \theta
$$

In closing this section, it is useful to verify that Corollary 6.5 and Theorem 6.1 give the same result for $\Delta r$. Let the projection of $\mathcal{A}$ onto the $\theta$ axis of $\mathfrak{B}$ be the closed interval $\left[\theta_{1}, \theta_{2}\right]$ so that $\mathcal{A}$ is the set of all points $(\theta, B)$ such that $\theta_{1} \leq \theta \leq \theta_{2}$ and $h(\theta) \leq B \leq m(\theta)$, where $h(\theta)$ and $m(\theta)$ are continuous functions forming the top and bottom portions of the boundary of $\mathcal{A}$. Using this in Corollary 6.5 yields

$$
\begin{aligned}
& \Delta r=-\gamma \oint_{C} B \sin 2 \theta \sec ^{2}(\theta-\varphi) d \theta \\
& \quad=-\gamma\left[\int_{\theta_{1}}^{\theta_{2}} h(\theta) \sin 2 \theta \sec ^{2}(\theta-\varphi) d \theta+\int_{\theta_{2}}^{\theta_{1}} m(\theta) \sin 2 \theta \sec ^{2}(\theta-\varphi) d \theta\right]
\end{aligned}
$$

or

$$
\Delta r=\gamma \int_{\theta_{1}}^{\theta_{2}}[m(\theta)-h(\theta)] \sin 2 \theta \sec ^{2}(\theta-\varphi) d \theta
$$

Applying this approach to Theorem 6.1 yields the same result, i.e., 


$$
\Delta r=-\iint_{A} F d \theta d B=\gamma \int_{\theta_{1}}^{\theta_{2}} \sin 2 \theta \sec ^{2}(\theta-\varphi) d \theta \int_{h(\theta)}^{m(\theta)} d B
$$

or

$$
\Delta r=\gamma \int_{\theta_{1}}^{\theta_{2}}[m(\theta)-h(\theta)] \sin 2 \theta \sec ^{2}(\theta-\varphi) d \theta
$$

\section{Conclusions}

Closed weak valued energy quantum systems have been shown to conform to a 1 st Law of Thermodynamics that is an extension of the usual 1st Law of Thermodynamics for closed mean energy quantum systems. In particular, the mathematical statement of the extended 1st Law not only includes the real valued heat and work exchange terms of the usual 1st Law, but it also contains additional complex valued heat and work exchange terms introduced by the AV energy gauge transformation that utilize energy uncertainty in their exchange processes. A peculiar unanticipated complex valued "pure" energy uncertainty exchange term that is required for weak valued energy conservation also appears in the mathematical statement of the extended 1st Law.

Application of the extended 1 st Law to a real valued spin-1/2 system in a uniform magnetic field revealed that the (real valued) heat and work exchange terms define a gauge potential and an associated non-vanishing gauge field which is produced by a changing gauge potential—or equivalently - by changes in the rates of heat and work exchange. It was also shown that the path integral of this gauge potential along a closed cycle $C$ in an appropriately parameterized plane and the integral of the gauge field flux through the area in the plane enclosed by $C$ are equal.

When this system is examined from the perspective of a principle fiber bundle with an appropriately parameterized plane as its base space and the additive group of real numbers as its typical fiber, the gauge potential was shown to define a "non-flat" connection on the bundle with the associated curvature specified by the gauge field. Interestingly, in this geometric setting, the path integral of the energy uncertainty exchange term along a closed cycle $C$ in this base space is such that the horizontal lift of $C$ does not close-i.e., it yields a geometric phase $\Delta r$ that is equal to (the negative of) this path integral. It was also shown that $\Delta r$ is also equal to the (negative) path integral of the gauge potential along $C$, as well as to the (negative) integral of the gauge field flux through the area enclosed by $C$.

In closing, it is noted that the results of this paper pose several open questions: (1) How can a closed quantum system be prepared so that its energy is weak valued? (2) What are the physical meanings of imaginary heat, work, and energy uncertainty exchange in a thermodynamic system? and (3) Does the principle fiber bundle model used in this paper and its consequences reflect physical reality?

The first question has been partially answered for the special case of the weak energy of evolution which is generated during the measurement of weak values when the PPS states are time dependent (for further detail the reader is referred to [31]). A more general answer to this question is currently under investigation by the author. Clues to answering the second question may reside in how the imaginary part of the weak energy of evolution affects the weak value measurement process [31] and the role it plays in the weak energy stationary action principle [32]. Although question (3) is more of a philosophical question than the other two, it is possible that the geometric phase $\Delta r$ exists physically and can be observed experimentally. Although the existence of $\Delta r$ is somewhat supported by the 
theoretical findings in [33] and [34], making such a measurement will likely depend upon finding a satisfactory answer to question (1). Nonetheless, a philosophical argument has been made to support the physical reality of the fiber bundle model presented in this paper. Specifically, Guttmann and Lyre [35] point out that the very successful "quantum gauge theories and gravitation give rise to fiber bundles with non-flat connections". They also suggest that "the existence of non-flat connections signifies the physical non-triviality of the fiber bundles involved" and believe that "in those cases involving bundles with non-flat connections, the fiber bundle formulations are to be taken seriously".

\section{Acknowledgments}

This work was supported by a grant from the Naval Surface Warfare Center Dahlgren Division's In-house Laboratory Independent Research Program. The author thanks the journal's referees for reading this manuscript and noting several areas where the extended 1st Law might be applicable. Additional thanks is given to John E. Gray for providing an initial critical reading the manuscript.

\section{Conflicts of Interest}

The author declares no conflict of interest.

\section{References}

1. Reznik, B. Interaction with a pre and post selected environment and recoherence. Available online: http://arxiv.org/abs/quant-ph/9501023 (accessed on 10 February 2005).

2. Kwiat, P.; Steinberg, A.; Chiao, R. Observation of a "quantum eraser": A revival of coherence in a two-photon interference experiment. Phys. Rev. A 1992, 45, 7729-7739.

3. Wong, H.; Cheng, K.; Chi, M. The quantum geometric phase between orthogonal states. Phys. Rev. Lett. 2005, 94, doi:10.1103/PhysRevLett.94.070406.

4. Hils, B.; Dultz, W.; Matrienssen, W. Nonlinearity of Pancharatnam's geometric phase in polarizing interferometers. Phys. Rev. E 1999, 60, 2322-2329.

5. Aharonov, Y.; Bergmann, P.; Lebowitz, J. Time symmetry in the quantum process of measurement. Phys. Rev. 1964, 134, doi:10.1103/PhysRev.134.B1410.

6. Aharonov, Y.; Albert, D.; Casher, A.; Vaidman, L. Novel properties of preselected and postselected ensembles. In New Techniques and Ideas in Quantum Measurement Theory; Greenberger, D., Ed.; New York Academy of Sciences: New York, NY, USA, 1986; pp. 417-421.

7. Aharonov, Y.; Albert, D.; Vaidman, L. How the result of a measurement of a component of the spin of a spin-1/2 particle can turn out to be 100. Phys. Rev. Lett. 1988, 60, 1351-1354.

8. Aharonov, Y.; Vaidman, L. Properties of a quantum system during the time interval between two measurements. Phys. Rev. A 1990, 41, 11-20.

9. Ritchie, N.; Storey, J.; Hulet, R. Realization of a measurement of a "weak value". Phys. Rev. Lett. 1991, 66, 1107-1110.

10. Parks, A.; Cullin, D.; Stoudt, D. Observation and measurement of an optical Aharonov-Albert-Vaidman effect. Proc. R. Soc. Lond. A 1998, 454, 2997-3008. 
11. Wang, Q.; Sun, F.; Zhang, Y.; Jian, L.; Huang, Y.; Guo, G. Experimental demonstration of a method to realize weak measurement of the arrival time of a single photon. Phys. Rev. A 2006, 73, doi: 10.1103/PhysRevA.73.023814.

12. Hosten, O.; Kwiat, P. Observation of the spin Hall effect in light via weak measurements. Science 2008, 319, 787-790.

13. Dixon, P.; Starling, D.; Jordan, A.; Howell, J. Ultrasensitive beam deflection measurement via interferometric weak value amplification. Phys. Rev. Lett. 2009, 102, doi:10.1103/PhysRevLett.102.173601.

14. Lin, B.; Chen, J. Performance analysis of an irreversible quantum heat engine working with harmonic oscillators. Phys. Rev. E 2003, 67, doi:10.1103/PhysRevE.67.046105.

15. Kieu, T. The second law, Maxwell's demon, and work derivable from quantum heat engines. Phys. Rev. Lett. 2004, 93, doi:10.1103/PhysRevLett.93.140403.

16. Rezek, Y.; Kosloff, R. Irreversible performance of a quantum harmonic heat engine. New. J. Phys. 2006, 8, doi:10.1088/1367-2630/8/5/083.

17. Zhang, T.; Liu, W.; Chen, P.; Li, C. Four-level entangled quantum heat engines. Phys. Rev. A 2007, 75, doi:10.1103/PhysRevA.75.062102.

18. Abe, S. Maximum-power quantum-mechanical Carnot engine. Phys. Rev. E 2011, 83, doi:10.1103/PhysRevE.83.041117.

19. Binder, F.; Vinjanampathy, S.; Modi, K.; Goold, J. Operational thermodynamics of open quantum systems. Available online: http://arxiv.org/abs/1406.2801 (accessed on 18 June 2014).

20. Vedral, V. Using temporal entanglement to perform thermodynamical work. Available online: http://arxiv.org/abs/1204.5559 (accessed on 27 April 2012).

21. Mann, R.; Martin-Martinez, E. Quantum thermometry. Found. Phys. 2014, 44, 492-511.

22. Brody, D.; Hughston, L. Quantum heat bath. Available online: http://arxiv.org/abs/1406.5780 (accessed on 24 June 2014).

23. Kosloff, R.; Geva, E.; Gordon, J. Quantum refrigerators in quest of the absolute zero. J. Appl. Phys. 2000, 87, 8093-8097.

24. He, J.; Chen, J.; Hua, B. Quantum refrigeration cycles using spin-1/2 systems as the working substance. Phys. Rev. E 2000, 65, 036145.

25. Ansari, M.; Nazarov, Y. Rényi entropy flows from quantum heat engines. 2015, 91, doi:10.1103/PhysRevB.91.104303.

26. Linden, N.; Popescu, S.; Skrzypczyk, P. The smallest possible heat engines. Available online: http://arxiv.org/abs/1010.6029 (accessed on 2 September 2014).

27. Frenzel, M.; Jennings, D.; Rudolph, T. Pure qubit work extraction revisited. Available online: http://arxiv.org/abs/1406.3937 (accessed on 3 November 2014).

28. Erez, N.; Gordon, G.; Nest, M.; Kurizki, G. Thermodynamic control by frequent quantum measurements. Nature 2008, 452, 724-727.

29. Parks, A. Weak values and the Aharonov-Vaidman gauge. J. Phys. A: Math. Theor. 2012, 43, doi:10.1088/1751-8113/43/3/035305.

30. Quan, H.; Liu, Y.; Sun, C.; Nori, F. Quantum thermodynamic cycles and quantum heat engines. Phys. Rev. E 2007, 76, doi:10.1103/PhysRevE.76.031105. 
31. Parks, A. Time-dependent weak values and their intrinsic phases of evolution. J. Phys. A: Math. Theor. 2008, 41, doi:10.1088/1751-8113/41/33/335305.

32. Parks, A. A weak energy stationary action principle for quantum state evolution. J. Phys. A: Math. Gen. 2003, 36, 7185-7192.

33. Parks, A. Pointed weak energy and quantum state evolution in Pancharatnam-Fubini-Study configuration space. J. Phys. A: Math. Gen. 2006, 39, 601-615.

34. Parks, A. Pointed weak energy and quantum geometric phase. J. Phys. A: Math. Theor. 2007, 40, 2137-2146.

35. Guttmann, Y.; Lyre, H. Fiber bundle gauge theories and "Field's Dilemma". Available online: http://arxiv.org/abs/physics/0005051 (accessed on 8 December 2009).

(C) 2015 by the authors; licensee MDPI, Basel, Switzerland. This article is an open access article distributed under the terms and conditions of the Creative Commons Attribution license (http://creativecommons.org/licenses/by/4.0/). 